\title{
Treatment of acute grade III acromioclavicular dislocation: a lack of evidence
}

\author{
E. Ceccarelli $\cdot$ R. Bondì $\cdot$ F. Alviti $\cdot$ R. Garofalo $\cdot$ \\ F. Miulli $\cdot$ R. Padua
}

Received: 20 March 2008/Accepted: 2 May 2008/Published online: 22 May 2008

(C) Springer-Verlag 2008

\begin{abstract}
Background Although nonoperative treatment is considered the standard of care for the treatment of grade I and II acromioclavicular joint injuries, the treatment of grade III injuries is controversial. There are as many methods of nonoperative treatment as there are for operative stabilization. That is why we conducted a literature research to find out the best evidence regarding the treatment of acute grade III acromioclavicular dislocation.

Method The research was limited to RCTs, systematic review and meta-analysis in the most representative databases. Even if research identifies more than 600 articles, only five were included in the study because there were RCTs, and systematic reviews, but no meta-analysis articles were found. Moreover, no meta-analysis was
\end{abstract}

E. Ceccarelli $\cdot$ R. Bondì $\cdot$ R. Padua $(\bowtie)$

Orthopedics Department, S. Giacomo Hospital,

Via E. Gianturco 4, 00196 Rome, Italy

e-mail: roberto.padua@fastwebnet.it

E. Ceccarelli $\cdot$ R. Bondì $\cdot$ R. Padua

GLOBE Evidence Based Orthopedics Working Group of Italian

Society of Orthopedics and Traumatology, Rome, Italy

F. Alviti

Department of Human Physiology and Pharmacology,

School of Specialization in Sports Medicine,

University "La Sapienza” of Rome, Rome, Italy

R. Garofalo

Ente Ecclesiastico Ospedale Generale Regionale,

Acquaviva delle Fonti, Bari, Italy

F. Miulli · R. Padua

Don Gnocchi Foundation, Rome, Italy

R. Padua

Nicola's Foudation, Arezzo, Italy performed because of differences of data published in the three RCTs (different type of surgical treatments and different outcome measures).

Results From the literature evaluation, clinical results seem to be comparable between the operative and the conservative treatments, but complications are more evident in the surgery group. Since there is not a preponderance of positive papers showing the benefits of a surgical technique over conservative therapy, the nonoperative treatment is still considered a valid procedure in the grade III acromioclavicular separation.

Conclusion More prospective randomized studies using validated outcome measures are needed to identify the suitable operation techniques for the acute injuries.

Keywords Acromioclavicular joint · Injuries .

Dislocations $\cdot$ RCTs $\cdot$ Systematic review $\cdot$ Meta-analysis

\section{Introduction}

Nonoperative treatment is considered the standard of care in case of grade I and II acromioclavicular joint dislocation, but the treatment of acute grade III is still controversial. Such injuries, classified by Allman [1] and Tossy et al. [2] as grade III, are characterized by the displacement of the outer end of the clavicle of one clavicular diameter or $1 \mathrm{~cm}$ on the anteroposterior radiograph. With respect to grade I and II injuries, characterized by rupture of the acromioclavicular ligaments with loss of horizontal stability, in grade III there is also the rupture of the coracoclavicular ligaments with displacement of the lateral clavicle with loss of vertical stability, producing a complete dislocation [3]. Surgery has been advocated to restore the anatomy of acromioclavicular joint but carries a 
significant risk of complications [4]. Instead, conservative treatment, even if does not restore the anatomy, allows patients to rehabilitate more rapidly [4-6]. Even if, in the last years, the number of publications about the surgical procedure are increasing, it is still not evident which is the gold standard and the conservative treatment is considered a valid procedure also for grade III acromioclavicular separation. The purpose of this study is to review the literature to determine the most adequate method of treatment for the acute dislocation and for its application in the clinical decision-making.

\section{Materials and methods}

A literature research limited to randomized clinical trials (RCTs), systematic reviews and meta-analyses publications was conducted in the most representative databases available: CochraneLibrary, Health Technology Asseement (HTA), TRIP, Medline, CINAHL and EMBASE. The following were the keywords for the search, identified through the National Library of Medicine's medical subject healing (MeSH) database: acromioclavicular joint injuries, acromioclavicular joint dislocations. We also conducted the research in the title and abstract fields with all possible combinations of these and other terms, such as: Tossy or Allman Type III dislocation, Rockwood Type III dislocation $[1,2]$, surgical therapies, and conservative therapies.

\section{Results}

The research identifies more than 640 articles regarding acromioclavicular dislocation, but only five articles were included in the study. Most of the articles were Level IV studies describing various nonoperative and operative methods of treating Grade III acromioclavicular separation and most of those, which compare various forms of operative stabilization versus nonoperative methods, were retrospective except for three, which were prospective RCT and two of them were a systematic review, but no metaanalyses publication were founded.

Imatani et al. [7] published a prospective randomized study comparing nonoperative treatment to two different forms of operative treatment. Patients were randomized by alternating treatment options based on time of presentation. Surgery techniques consisted in an open reduction and stabilization with Steinman pins placed across the acromioclavicular joint or stabilization with a coracoclavicular screw; the fixation device was removed at 3 months after surgical procedure, while the nonoperative treatment consisted of a sling for 3 weeks. Patients were assessed at 1 year radiographically, clinically and with a custom 100-point outcome measure that included pain, function and motion. Bias of the study was the relatively low number of patients ( 11 operative and 12 nonoperative) and the use of a nonvalidated custom outcome measure. The authors concluded that surgical stabilization was no better than nonoperative treatment and recommended all acute complete acromioclavicular separation be treated nonoperatively.

Larsen et al. [4] prospectively randomized 84 patients via sealed envelopes to non operative management or modified Phemister procedure [4]. Nonoperative treatment consisted of placement in a sling for 4 weeks and physiotherapy; operative treatment consisted of open reduction and repair of the acromioclavicular and coracoclavicular ligaments with temporary acromioclavicular pinning, removed at 5-12 weeks after the procedure. Patients were assessed at 3 and 13 months radiographically and with a 12-point custom scoring system that included pain, motion and strength. Results at 3 months were better in the nonoperative group, but there was no differnce at 13 months. Radiographically, all but two of the operative patients maintained the reduction, whereas all patients in the nonoperative group continue to have residual displacement. As complications he reported 6 superficial infections in the operative group and pin migration or breakage in 21 patients. Two patients of the operative group and three in the nonoperative had and additional distal clavicular excision for the residual pain. The authors concluded that most patients could be treated nonoperatively with a shorter rehabilitation time. They also suggested that thin patients and those who perform heavy or overhead works be considered for operative stabilization based on subjective complaints.

In the last prospective study, reported by Bannister et al. [5], 60 patients were randomized via drawn numbers to nonoperative treatment or operative stabilization with a Bosworth method [5]. Patients were treated nonoperatively with a sling for 2 weeks and then they participated in a rehabilitation program, or were treated operatively with coracoclavicular screw fixation and then were engaged in the same rehabilitation program. The screws were removed after 6 weeks. Patients were evaluated after 4 years through the Imatani's scoring system. In the nonoperative group, four patients failed to respond to treatment and underwent surgery for weakness or pain. As regards complications, in the operative group, there were an overall of 45 cases including loss of reduction and hardware failure. Also in this study authors concluded that nonoperative treatment was superior except in cases of severe displacement.

The literature review published by Phillips et al. [8] is mentioned by database of abstracts of reviews of effects (DARE). It was performed to clarify available information which influences decision whether to advise a young adult 
patient to undergo surgery for a severely displaced acromioclavicular dislocation. Relevant articles were retrieved using several different search strategies within Medline from 1966 to 1997, and references quoted in the papers retrieved also were screened for relevance. They found more than 600 articles published on the acromioclavicular dislocation in the English language listed in the Index Medicus. Only articles that describe severely displaced dislocation were selected and this largely meant Tossy et al. or Allman Type III and Rockwood et al. Type III dislocation. Only four papers contained overall outcome data on patients treated surgically and conservatively and only two of them were randomized. These two papers give a cumulative rate of satisfactory surgical outcome of $90 \%$ and of satisfactory conservative outcome of $91 \%$. A subgroup analysis was performed as regards pain, range of movement, power and complications. In particular, the meta-analysis reveals that there was a persistent deformity in the conservative group instead a low rate of deformity with surgical treatment. Meanwhile infection and the need of additional surgery were more frequent in the surgery group. No significant difference has been detected between the two groups as regarding the satisfactory outcome.

Also the systematic review published by Spencer [9] in 2006 was pursued to determine if grade III acromioclavicular joint separations are best treated operatively or nonoperatively. It was performed in the English-language literature using the Medline and the EMBASE database. The initial search resulted in 469 references, where 56 of these met the inclusion criteria of pertaining to Grade III separations. Only nine studies met the inclusion criteria of comparing the results of nonoperative treatment to various forms of operative stabilization. Three of these studies were prospective and randomized, whereas the others were retrospective analysis. The author pointed out that the results must be interpreted carefully because they are specific to the particular type of operative stabilization and are not necessarily applicable to other forms of operative procedures. Moreover he pointed out the impossibility of performing a meta-analysis because of the different outcome measures and procedures used in publications. That is why he gave an accurate description of prospective and nonprospective papers in a chronologic order, underlying the limitation of the study due to the lack of validated outcome measures, and the use of different surgical procedures that are seldom used today. All the three randomized prospective studies concluded that nonoperative treatment was superior because the surgical results were no better and were associated with more complications. In addition, surgical procedures were associated with increased convalescence time, away from work and sport. The only adverse result of the conservative treatment seems to be the persistence of the dislocation of the acromioclavicular joint even if it is not correlated to worst clinical outcomes and it could be present also in the surgery group after device removal even if in a lower percentage. Despite the limitations of this systematic review the author concludes that nonoperative treatment seems to be superior, till now, to surgical approach in the treatment of acute acromioclavicular severe dislocation.

\section{Discussion}

Although nonoperative treatment is the gold standard for grade I and II acromiomioclavicular joint dislocation, there are controversial opinions regarding grade III acrmioclavicular dislocation [4, 5, 7-9], and it is also evidenced by the increasing number, in the last years, of articles regarding new techniques of treatment. The purpose of this study was to review the literature, following evidence-based medicine principles, to find out the best evidence regarding the treatment of acute grade III acromioclavicular separation. But the insufficient number of RCTs, or complete systematic review and the absence of meta-analysis biased against the research. More over none of the studies evaluated used validated outcome measures and the data were not complete; different surgical procedures were performed and some of them are not routinely used today [9]. That is why the comparison of the results of the few RCTs is difficult and no metaanalysis evaluation was done. What we can say is that there is no preponderance of positive papers showing the benefits of a surgical technique over conservative therapy [8]. Even if the clinical results seem to be comparable as regards pain relief, range of motion and strength and complications are more evident in the surgery group than in the conservative group [5, 8, 9]. The operation should be justified as a cosmetic procedure, whereas conservative treatment frequently results in a persistent deformity. Some authors suggest surgery for patients who perform heavy or overhead work, but new research has to be done in this direction to confirm the advantage of anatomical reduction in such patients [4].

Despite the limitation of these reviews we can say that there does not seem to be any reason to recommend an operative procedure to a patient with an acute Allman [1] or Tossy et al. [2] type III injury and more research is needed to identify the suitable operation techniques for the acute and severe acromioclavicular dislocation. However, the choice must be discussed and let to the patient himself. Moreover, to perform a meta-analysis evaluation more prospective randomized studies using validated outcome measures are needed and future studies should include contemporary operative methods and seek to stratify certain populations that place high demands on the shoulder. 
Acknowledgments Conflict of interest statement The authors declare that they have no conflict of interest related to the publication of this manuscript.

\section{References}

1. Allman FLJ (1967) Fractures and ligamentous injuries of the clavicle and its articulation. J Bone Joint Surg 49A:774-784

2. Tossy JD, Mead NC, Sigmond HM (1963) Acromio-clavicular separation: useful and practical classification for treatment. Clin Orthop 28:111-119

3. Trikha SP, Acton A, Wilson AJ et al (2004) A new mwthod of arthroscopic reconstruction of the dislocated acromio-clavicular joint. Ann R Coll Surg Engl 86:161-164
4. Larsen E, Bjerg-Nielsen A, Cristensen P (1986) Conservative or surgical treatment of acromioclavicular dislocation. J Bone Joint Surg Am 68:552-555

5. Bannister GC, Wallace WA, Stableforth PG et al (1989) The menagement of acute acromioclavicular dislocation. J Bone Joint Surg Br 71:848-850

6. Galpin RD, Hawkins RJ, Grainger RW (1985) A comparative analysis of operation versus nonoperative treatment of grade III acromio-clavicular separations. Clin Orthop 193:150-155

7. Imatani RJ, Hanlon JJ, Cady GW (1975) Acute complete acromioclavicular separation. J Bone Joint Surg Am 57:328-332

8. Phillips AM, Smart C, Groom AFG (1998) Acromioclavicular dislocation. Conservative or sugical therapy. Clin Orthop 353:10-17

9. Spencer EE (2006) Treatment of grade III acromioclavicular joint injuries. Clin Orthop 455:38-44 